\title{
Análise cienciométrica de espaços verdes urbanos e seus serviços ecossistêmicos
}

\author{
Scientific analysis of urban green spaces and its ecosystem services
}

\section{Análisis cienciométrica de espacios verdes urbanos y sus servicios ecosistémicos}

\author{
Fabiany Sampaio Bertucci Tavares ${ }^{1}$ \\ Ivan Bergier ${ }^{2}$ \\ Eliane Guaraldo ${ }^{1}$
}

Recebido em 31/05/2019; revisado e aprovado em 10/11/2019; aceito em 19/11/2019

DOI: http://dx.doi.org/10.20435/inter.v22i1.2596

\begin{abstract}
Resumo: O acelerado crescimento populacional e urbano nas últimas décadas intensificou a necessidade de pesquisas sobre o ecossistema urbano e os serviços por ele oferecidos, visando ao bem-estar da população urbana por meio da conservação da natureza. O reconhecimento dos serviços ecossistêmicos fornecidos pelos Espaços Verdes Urbanos (EVUs) pode subsidiar a tomada de decisão para que as cidades promovam o desenvolvimento sustentável, conservando e/ou restaurando os serviços ecossistêmicos e a biodiversidade, para que a vida das pessoas nas cidades seja viável diante dos desafios impostos pelas mudanças climáticas. Este artigo tem por objetivo principal avaliar como os serviços ecossistêmicos fornecidos pelos EVUs estão sendo abordados no meio científico. Realizou-se um levantamento cienciométrico e, posteriormente, uma análise bibliográfica dos principais artigos para avaliar o conhecimento científico atual, as principais limitações teóricas e as lacunas e oportunidades para avanços no conhecimento sobre o tema. Os resultados apontam que as pesquisas dos serviços ecossistêmicos em EVUs foram intensificadas a partir de 2005 e apresentaram uma taxa exponencial de crescimento de $30 \%$ ao ano, particularmente nos países mais desenvolvidos. A análise bibliográfica aponta que os serviços ecossistêmicos reguladores e culturais no contexto urbano são aqueles de maior importância, e que são necessárias novas metodologias para avaliar e eventualmente precificar esses serviços.
\end{abstract}

Palavras-chave: eventos extremos; estoque de carbono; evapotranspiração, infraestrutura verde; serviços ecossistêmicos.

\begin{abstract}
The accelerated urban and population growth in the last decades has intensified the need for research about urban ecosystem and their services associated with the urban population's well-being through nature conservation. The acknowledgment of ecosystem services provided by Urban Green Spaces (UGSs) can support decision-making to promote urban sustainable development, therefore conserving and/or restoring ecosystem services and biodiversity aiming to adapt urban people to the forthcoming challenges of climate change. This article aims to assess how ecosystem services provided by UGSs have been evolved in the scientific community. A scientometric survey and a bibliographical analysis of the main articles helped to assess the current scientific knowledge, the main theoretical limitations and gaps, as well as the opportunities for advancing the knowledge on this subject. Results indicate that researches on ecosystem services in UGSs increased since 2005 at an annual exponential growth rate of 30\%, especially in more developed countries. Bibliographical analysis points out that both regulatory and cultural ecosystem services in urban context are those which has been attributed higher importance, and that new methodologies are necessary to assess and possibly to value these services.
\end{abstract}

Keywords: extreme events; carbon stock; evapotranspiration, green Infrastructure; ecosystem services.

Resumen: La población acelerada y el crecimiento urbano en las últimas décadas intensificaron la necesidad de la investigación sobre el ecosistema urbano y los servicios por ello proporcionados, teniendo como objetivo el bienestar de la población urbana por medio de la conservación de la naturaleza. El reconocimiento de los servicios ecosistémicos proveídos por Espacios Verdes Urbanos (EVUs) puede subvencionar la toma de decisión de modo que las ciudades promuevan el desarrollo sostenible, la conservación y/o la restauración de los servicios ecosistémicos de la biodiversidad, de modo que la vida de la gente en las ciudades sea viable delante de los desafíos impuestos por los cambios climáticos. Este artículo tiene como el objetivo principal evaluar como los servicios ecosistémicos proveídos por el EVU están siendo abordados en el

\footnotetext{
${ }^{1}$ Universidade Federal de Mato Grosso do Sul (UFMS), Campo Grande, Mato Grosso do Sul, Brasil.

2 Empresa Brasileira de Pesquisa Agropecuária (Embrapa), Corumbá, Mato Grosso do Sul, Brasil.
} 
medio científico. Se realizó una encuesta sobre el cienciometrico y, después, un análisis bibliográfico de los principales artículos para evaluar más adelante el conocimiento científico actual, las limitaciones teóricas principales, los huecos y las ocasiones para los avances en el conocimiento en el tema. Los resultados señalan más que la investigación de los servicios ecosistémicos en EVUs había sido intensificada a partir de 2005 y había presentado un impuesto exponencial del crecimiento en el 30\% al año, particularmente en los países desarrollados. El análisis bibliográfico señala que los servicios ecosistémicos regulatorios y culturales en el contexto urbano son los de mayor importancia, y que son necesarias nuevas metodologías para evaluar y eventualmente, cotizar estos servicios..

Palabras claves: eventos extremos; fuente del carbón; evapotranspiración, infraestructura verde; servicios ecosistémicos.

\section{INTRODUÇÃO}

O aumento da população urbana mundial e a crescente urbanização têm levado à expansão do espaço construído, tornando os espaços verdes escassos e segregados. Diante dessa realidade, o termo "ecossistema urbano" tem ganhado destaque em pesquisas acadêmicas, sendo considerado um conjunto diversificado de habitats, incluindo 1) espaços verdes, como parques, florestas urbanas, jardins, pátios e áreas de campus; e 2) espaços azuis, incluindo riachos, lagos, lagoas, valas artificiais e lagoas de retenção de águas pluviais (BOLUND; HUNHAMMAR, 1999; GÓMEZ-BAGGETHUN; BARTON, 2013; ELMQVIST et al., 2015). Os espaços verdes são um dos mais importantes produtores de serviços ecossistêmicos no contexto urbano (WOLCH, J. R.; BYRNE, J.; NEWELL, J. P., 2014) e estão intimamente ligados ao bem-estar humano, no que diz respeito à sua diversidade e qualidade (NIEMELA, 2014).

Nas políticas públicas, os ecossistemas urbanos podem ser retratados também como "infraestruturas verdes" e, de forma crescente, têm feito parte de debates políticos. Porém, ao comparar com outras áreas, a atenção para os serviços ecossistêmicos na área urbana ainda tem sido muito modesta (GÓMEZ-BAGGETHUN; BARTON, 2013; DAVIES et al., 2013). Isto porque a importância de alguns serviços ecossistêmicos e os seus impactos podem ser percebidos globalmente, como é o caso do sequestro e armazenamento de carbono. Já outros, como a regulação do microclima, evapotranspiração, regulação do ruído e os serviços culturais, são mais bem percebidos localmente (NIEMELA et al., 2010).

O conceito utilizado hoje para o termo "serviços ecossistêmicos" é: os benefícios que as pessoas obtêm dos ecossistemas (MILLENNIUM ECOSYSTEM ASSESSMENT [MEA], 2005). Antes disso, os serviços ecossistêmicos eram definidos como "os benefícios que as populações humanas obtêm, direta ou indiretamente, das funções dos ecossistemas" (COSTANZA et al., 1997). Os serviços ecossistêmicos são classificados em: serviços de suporte, como formação do solo, fotossíntese e ciclagem de nutrientes; serviços de provisão, que são os produtos obtidos, como o fornecimento de alimentos, água e madeira; serviços reguladores, que são os benefícios que regulam as condições ambientais, condições climáticas, controle de doenças, resíduos e qualidade da água; e serviços culturais, que são os benefícios recreativo, estético e espiritual.

Com o objetivo principal de avaliar como os serviços ecossistêmicos fornecidos pelos EVUs estão sendo abordados no meio científico, o presente trabalho busca fazer o registro e a análise quantitativa dos artigos científicos que abordam o tema, bem como o levantamento bibliográfico para uma base teórica que permita avaliar as principais limitações e lacunas para pesquisas futuras. 


\section{MATERIAIS E MÉTODOS}

Para a triagem e organização do levantamento bibliográfico, foi utilizado o software EndNote, versão X5, que faz a importação das informações bibliográficas dos resultados das pesquisas feitas nas bases de dados, que foram: Web of Science, Scopus, ScienceDirect e SciELO.

Os strings utilizados para buscas nas bases de dados foram: "ecosystem service\$" OR "environmental service\$" OR "ecohydrology* service\$" AND forest OR "open space\$" OR "green space\$" AND "urban*". As buscas dos termos foram para presença nos títulos, palavras-chave ou resumos, utilizando os resultados de todo o período de tempo, desde a primeira publicação em cada base de dados, até a data da compilação, que foi no dia dois de maio de dois mil e dezoito.

O refinamento do resultado geral de publicações disponíveis nas bases de dados pesquisadas foi realizado em etapas. As etapas foram: eliminação de duplicidades; escolha dos títulos alinhados; escolha dos resumos alinhados; e escolha dos textos alinhados.

\section{RESULTADOS}

A partir da importação dos dados no EndNote, a quantidade total de publicações foi de 2.480 (dois mil quatrocentos e oitenta), passando para 1.615 (mil seiscentos e quinze) após a eliminação das duplicidades. Na segunda etapa, a verificação das publicações cujos títulos se alinhavam ao tema da pesquisa reduziu esse número para 526 (quinhentos e vinte e seis). Na terceira etapa, foram alinhadas publicações cujos resumos se vinculavam ao tema de interesse, chegando a um resultado de 113 (cento e treze) publicações. Com a última etapa, a escolha dos textos alinhados, o número de publicações se reduziu para 38 (trinta e oito).

Tabela 1 - Etapas de filtragem das publicações

\begin{tabular}{l|c}
\hline Etapa & N. de Publicações \\
\hline Todos os resultados & 2.480 \\
\hline Eliminação de duplicidades & 1.615 \\
\hline Escolha dos títulos alinhados & 526 \\
\hline Escolha dos resumos alinhados & 113 \\
\hline Escolha dos textos alinhados & 38 \\
\hline
\end{tabular}

Fonte: Os autores.

Ao analisar todos os resultados obtidos (2.480 publicações), é possível observar que as publicações se iniciaram no ano de 1987 e seu ritmo de aumento (total acumulado por ano) foi a uma taxa exponencial de aproximadamente $30 \%$ ao ano $\left(R^{2}=0.9796\right.$, ver Gráfico 1$)$. A partir de 2005, percebe-se que a frequência de publicações aumentou e permanece crescente a cada ano, demonstrando o interesse pelo tema nas pesquisas em todo o mundo (Gráfico 1). 
Gráfico 1 - Evolução exponencial (escala logarítmica na base 2) do número total de publicações, de acordo com os critérios escolhidos de busca

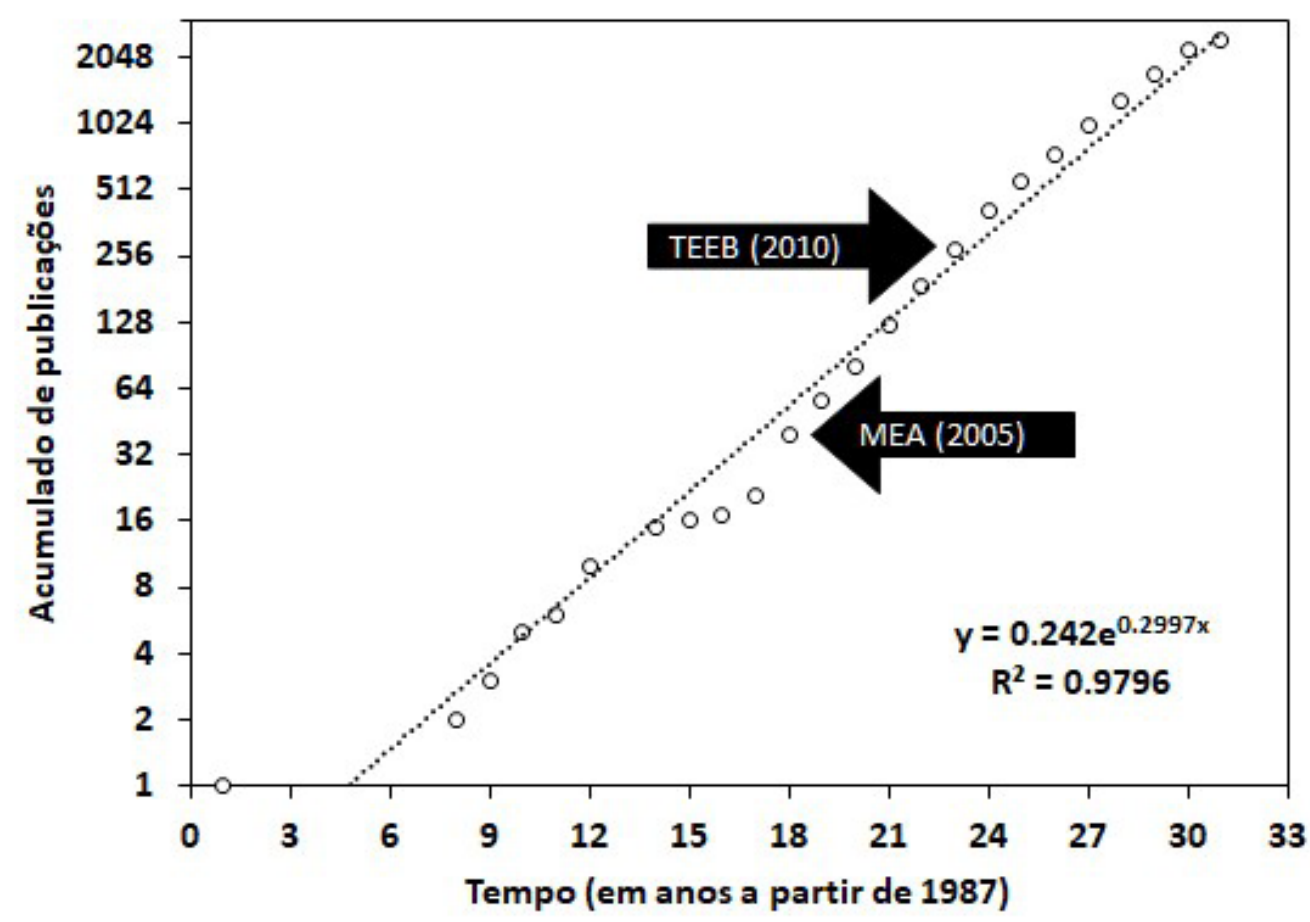

Fonte: Os autores.

O aumento do interesse pelos estudos de serviços ecossistêmicos em EVU foi certamente estimulado pela Millennium Ecosystem Assessment (MEA, 2005), uma avaliação científica das condições e tendências dos ecossistemas do mundo e dos serviços que eles fornecem. Outro possível fator pelo aumento do interesse do tema foram os estudos da The Economics of Ecosystems and Biodiversity (TEEB, 2009), sustentando que as cidades dependem dos ecossistemas nelas presentes para sustentar as condições de vida, e que a perda do ecossistema urbano envolve impactos sociais, culturais, econômicos e ambientais (GÓMEZ-BAGGETHUN; BARTON, 2013; NIEMELA et al., 2010).

Analisando as bases de dados, identificou-se que apenas 25 publicações (equivalente a $1 \%$ do resultado geral) são da base de dados brasileira SciELO (Gráfico 2), o que aponta que os tópicos são pouco abordados em revistas brasileiras. 
Gráfico 2 - Distribuição relativa das publicações pesquisadas nas bases de dados bibliográficos entre 1987 e 2018

\section{Distribuição das publicações nas bases avaliadas}

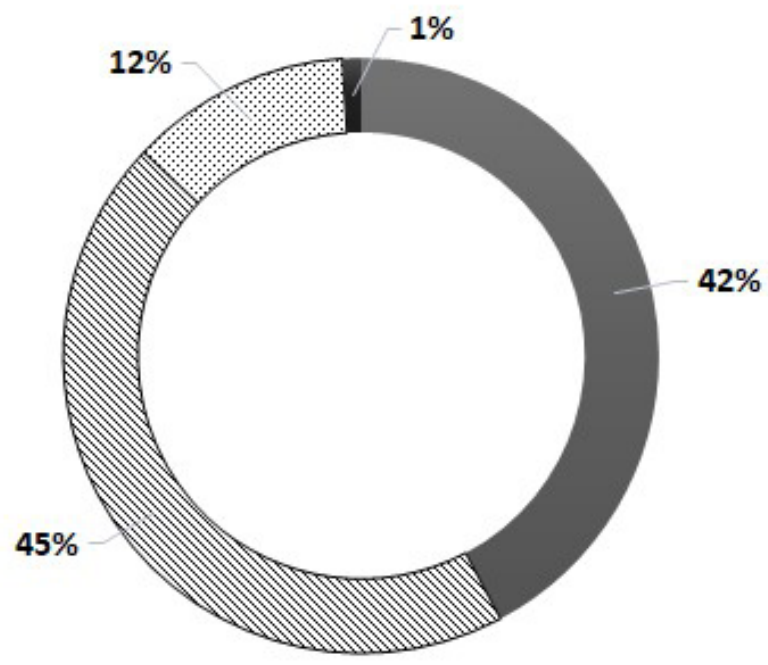

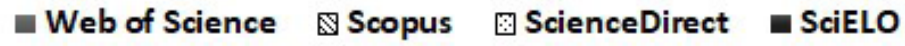

Fonte: Os autores.

A pesquisa dos strings apresentada em nuvem de tags (Imagem 1) mostrou que o termo "ecosystem services" prevalece sobre qualquer outro e revelou palavras-chave não pesquisadas que foram grandemente utilizadas nas pesquisas, como: "land use", "carbon", "climate change", "biodiversity", "landscape", "conservation", entre outras.

Imagem 1 - Nuvem de tags dos 30 termos (strings) mais frequentes em palavras-chave de todos os 1.615 artigos encontrados (sem duplicidade)

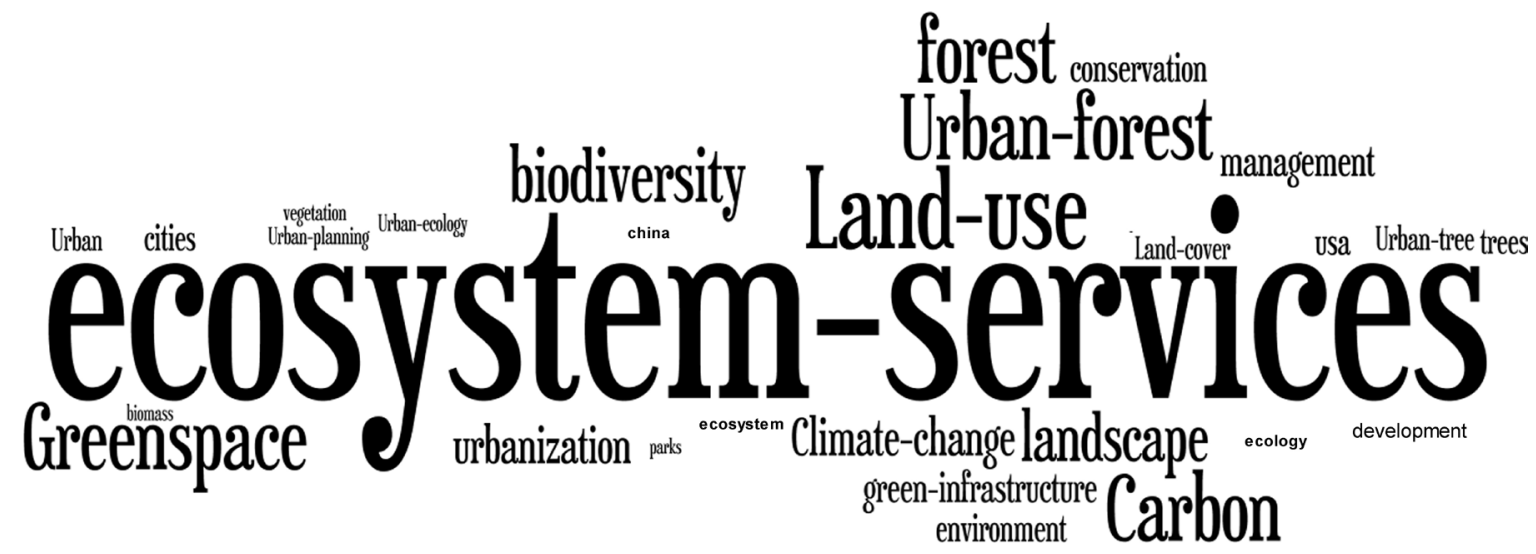

Fonte: Os autores. 


\section{DISCUSSÃO}

O foco da pesquisa sobre o espaço verde urbano e os serviços ecossistêmicos tem sido mais contundente nos países desenvolvidos, particularmente nos Estados Unidos, China, Alemanha, Suécia e Reino Unido (Gráfico 3). Poucos trabalhos foram encontrados na África, América do Sul ou Rússia. Entende-se, então, que os estudos dos EVUs e os serviços ecossistêmicos prestados por eles ainda precisam ser mais estudados e difundidos nos países em desenvolvimento, visando ampliar a abordagem interdisciplinar dos conhecimentos de ciências naturais, engenharias, arquitetura e sociais (KABISCH; QURESHI; HAASE, 2015).

Apesar do desenvolvimento de vários modelos para quantificar e valorizar os serviços ecossistêmicos urbanos na última década (ANDERSSON-SKÖLD et al, 2018), os trabalhos analisados apontam que ainda são necessárias mais pesquisas que envolvam o desenvolvimento de métodos apropriados para a quantificação dos serviços que os EVUs oferecem (HAASE et al., 2014; LUEDERITZ et al., 2015).

Gráfico 3 - As publicações mais alinhadas ao tema se concentram nos países mais desenvolvidos

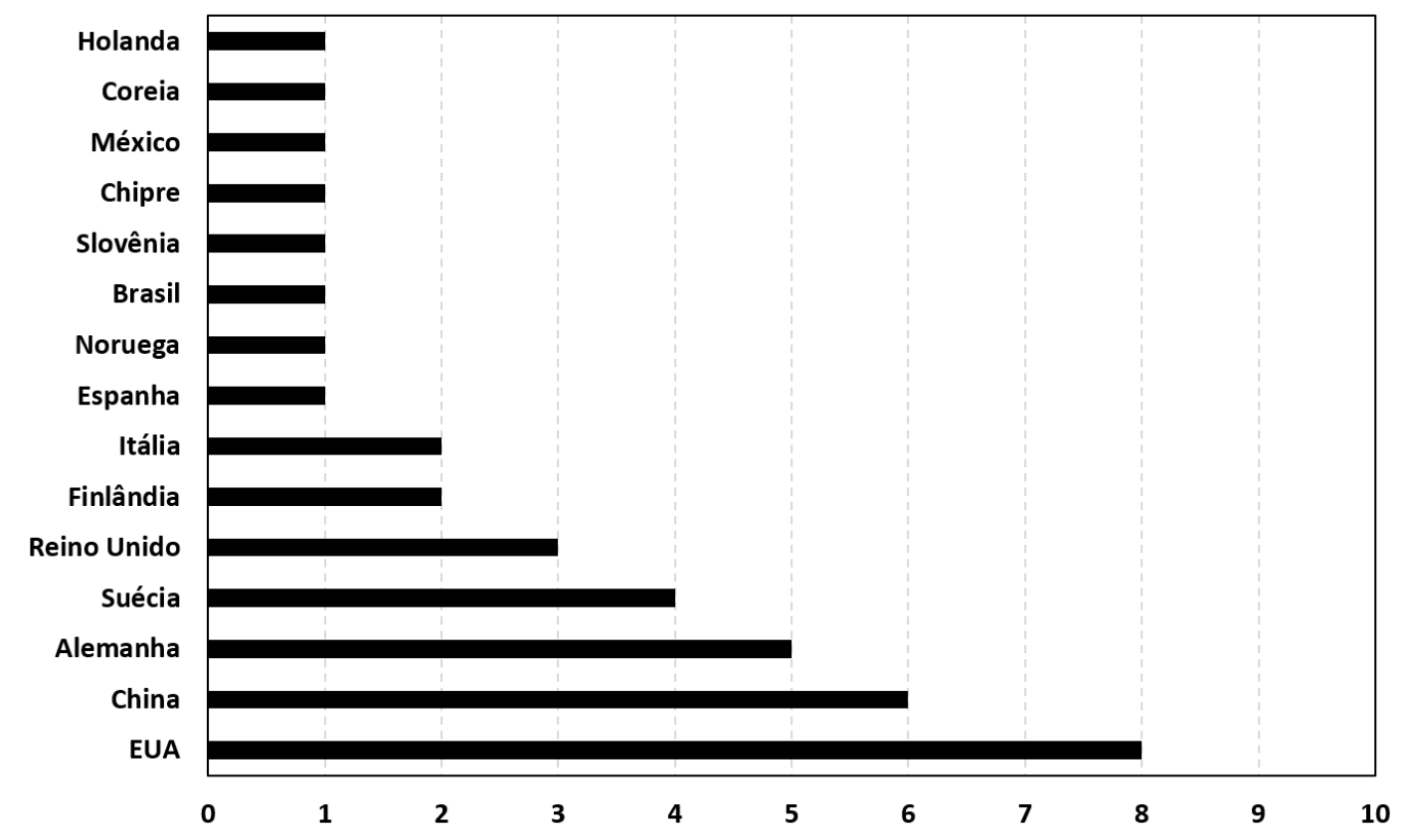

Fonte: Os autores.

As produções analisadas mostram que é necessário avaliar as características ambientais e socioeconômicas de cada local para encontrar quais serviços ecossistêmicos são mais relevantes em uma determinada cidade (GÓMEZ-BAGGETHUN; BARTON, 2013). Porém, em geral, os estudos apontam que, no contexto urbano, sobressaem-se os serviços ecossistêmicos reguladores $e$ culturais, visto a necessidade de purificação do ar, regulação do ruído, regulação (térmica) do microclima, drenagem de águas pluviais, tratamento de esgoto, recreação e as contribuições para a saúde mental e física da população (BOLUND; HUNHAMMAR, 1999; GÓMEZ-BAGGETHUN; BARTON, 2013; NIEMELA et al., 2010). 
Os Serviços reguladores são os benefícios que regulam as condições ambientais, condições climáticas, controle de doenças, resíduos e qualidade da água (MEA, 2005). A mitigação dos danos de desastres naturais, a preservação do habitat natural e da biodiversidade, a redução das águas pluviais, o resfriamento, entre outros, são apenas alguns exemplos dos serviços ecossistêmicos reguladores relevantes na área urbana (ESCOBEDO; KROEGER; WAGNER, 2011).

Quanto aos serviços reguladores, o estoque de carbono florestal (ECF) e o processo de evapotranspiração (ET) apresentam uma importância de destaque, visto que é a partir deles que outros serviços podem ser percebidos.

\subsection{Estoque de carbono florestal (ECF)}

O ECF apresenta grande importância na mitigação das mudanças climáticas (DAVIES et al, 2011; NOWAK et al., 2013) e, apesar da crescente urbanização, as florestas urbanas são frequentemente desconsideradas dos orçamentos de carbono, por não serem bem compreendidas ou quantificadas, ou simplesmente por serem subestimadas (NOWAK et al., 2013; DORENDORF et al., 2015).

As cidades consomem grande quantidade de energia, e as crescentes urbanização e industrialização fazem com que aumente a emissão e a concentração de dióxido de carbono $\left(\mathrm{CO}_{2}\right)$ na atmosfera (ESCOBEDO; KROEGER; WAGNER, 2011; DORENDORF et al., 2015). Visto isso, os espaços verdes urbanos são relevantes para a cidade, pois as árvores fixam o $\mathrm{CO}_{2}$ da atmosfera durante a fotossíntese e o armazenam em sua biomassa e nos solos, atuando como sumidouros de carbono nas cidades (NOWAK et al., 2013).

Reduzir emissão de $\mathrm{CO}_{2}$ para a atmosfera tem sido um grande desafio para os planejadores e gestores ambientais urbanos. Apesar dos estudos sobre ECF por espaços verdes urbanos estarem aumentando, estes têm se concentrado nos países mais desenvolvidos de zonas temperadas ou de florestas subtropicais (DAVIES et al., 2013).

A análise da literatura mostrou que a maioria das estimativas de carbono da vegetação urbana é feita a partir de equações alométricas (DAVIES et al., 2011; DAVIES et al., 2013; NOWAK et al., 2013). No entanto, todas essas equações são usualmente parametrizadas para cada espécie, dificultando a estimativa em áreas florestais diversificadas, além do fato de que, quando se trata de espaços verdes urbanos, os estudos se dão em sua maioria no hemisfério Norte.

\subsection{Evapotranspiração (ET)}

Outro serviço regulador de água de grande importância é a evapotranspiração, que tem sido denominada como "fluxo de água verde" (D'ODORICO et al., 2010) e faz parte da dinâmica da interação do ciclo da água com a biota, uma área de estudo conhecida como eco-hidrologia. Os fluxos interconectados e interdependentes de água verde (vegetação) e azul (rios) desempenham importantes funções ecológicas (imagem 2). 
Imagem 2 - Fluxos de água azul e verde do ciclo hidrológico.

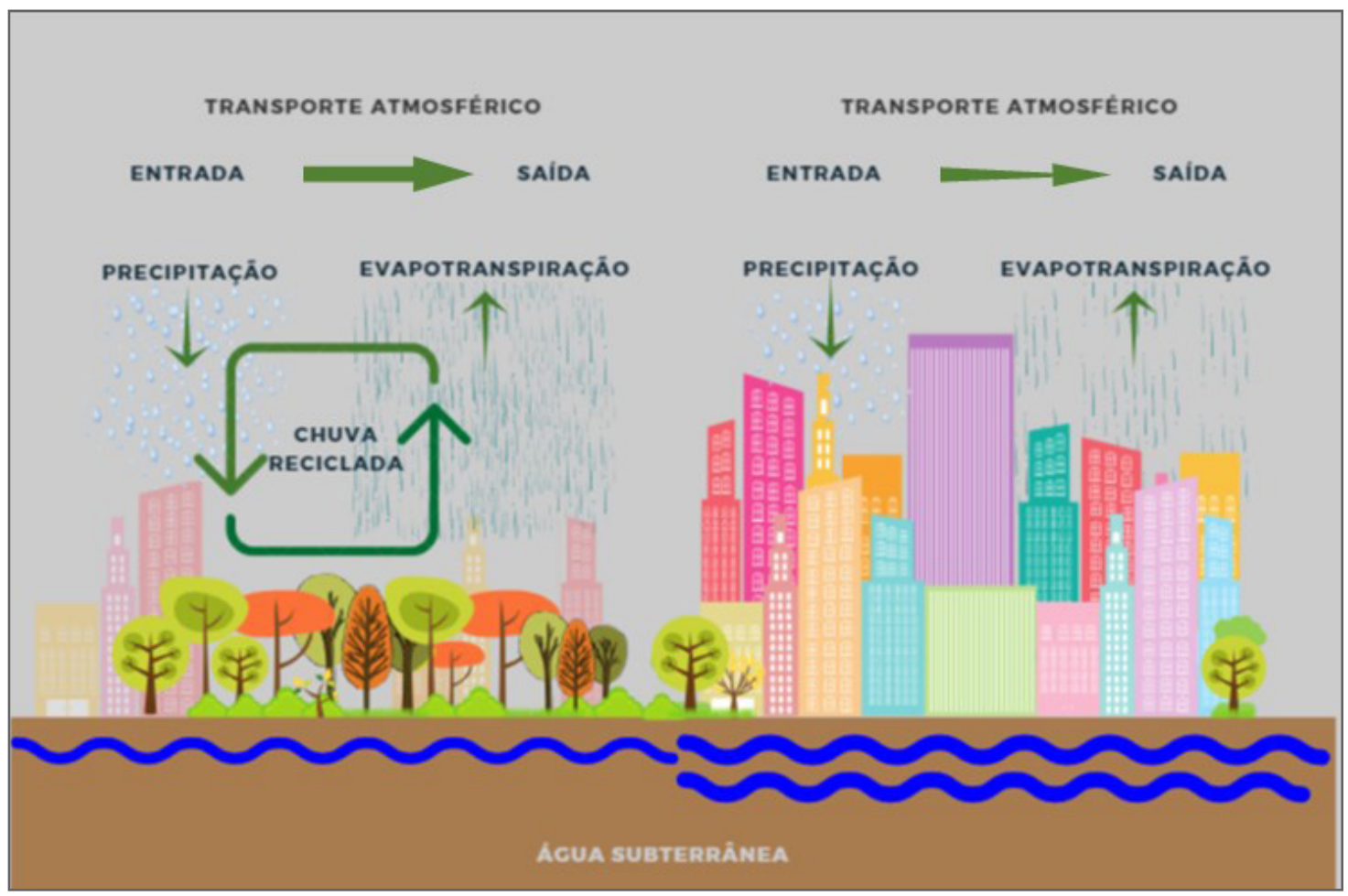

Fonte: Adaptação de Aragão (2012) pelos autores.

A vegetação é responsável pela recarga dos aquíferos e pela ET da água precipitada na forma de chuva. As mudanças no uso da terra, como o desmatamento e a urbanização, modificam a dinâmica dos processos eco-hidrológicos, levando a uma diminuição da infiltração nos solos, da recarga dos aquíferos e da ET, resultando em um aumento do escoamento superficial das águas pluviais (D'ODORICO et al., 2010).

Os EVUs, por meio da ET, influenciam também nos microclimas da cidade, podendo diminuir a temperatura na vizinhança, assim como aumentar a umidade do ar; além disso, filtram o ar poluído da cidade (DERKZEN et al., 2015). Essa mudança no microclima contribui para a diminuição das ilhas de calor que têm se formado nos centros urbanos. Embora a diminuição em termos absolutos pareça pequena, ela evita problemas de saúde pública relacionados ao calor excessivo e à exposição solar (COUTTS; RAHN, 2015).

\subsection{Lacunas científicas}

A pesquisa apontou lacunas sobre a produção científica relacionada aos serviços ecossistêmicos prestados por EVU. Em primeiro lugar, observou-se que a maior parte da produção ocorre em países desenvolvidos, com grande destaque para países da Europa, EUA e China, sendo zonas temperadas ou de florestas subtropicais. Pouca coisa foi encontrada em países da África ou América do Sul, e pesquisas brasileiras corresponderam a cerca de $1 \%$ do resultado geral. Espera-se que as pesquisas futuras sobre os temas sejam realizadas também em países em desenvolvimento, pois a maior conscientização sobre os serviços ecossistêmicos poderá contribuir para uma estrutura e design mais eficientes das cidades, assim como para maiores variedades de tipologias florestais. 
Outra observação é a falta de uma metodologia para avaliar e quantificar os serviços ecossistêmicos dos EVUs. No geral, quando se trata de ECF, utilizam-se equações alométricas da literatura para certas espécies, dificultando, assim, a análise de florestas diversas e em escalas variadas. A ET foi pouco abordada nos artigos analisados. Apesar da importância desse serviço para o bem-estar humano e para o ecossistema, não foi identificada uma metodologia clara para quantificá-lo.

\section{CONCLUSÃO}

As pesquisas científicas relacionadas aos serviços ecossistêmicos fornecidos pelos EVUs foram intensificadas a partir de 2005 e apresentam um crescimento de $37 \%$ por ano. Porém, o foco desses estudos está em países desenvolvidos. Os estudos analisados apontam que, no contexto urbano, os serviços ecossistêmicos reguladores e culturais são aqueles de maior importância, e que, entre os reguladores, o ECF e a ET são alguns dos principais serviços prestados por EVU.

Apesar da importância de tais serviços, poucas pesquisas mostram metodologias para avaliálos e quantificá-los. Diante dos desafios enfrentados no mundo contemporâneo, há necessidade de avançar pesquisas nessa linha de conhecimento que apoiem os gestores e tomadores de decisão nas áreas urbanas, no caminho do desenvolvimento equilibrado das cidades, conforme previsto pelos Objetivos de Desenvolvimento Sustentável (ODS): 1) Boa Saúde e Bem-Estar; 2) Redução das Desigualdades; 3) Cidades e Comunidades Sustentáveis; 4) Combate às Alterações Climáticas; e 5) Vida sobre a Terra das Nações Unidas.

\section{FINANCIAMENTO}

O financiamento para a conclusão deste estudo, vinculado ao Programa de Pós-Graduação em Recursos Naturais (PGRN) da Faculdade de Engenharias, Arquitetura e Urbanismo e Geografia da Universidade Federal de Mato Grosso do Sul (FAENG/UFMS), foi cedido pela Fundação de Apoio ao Desenvolvimento do Ensino, Ciência e Tecnologia do Estado de Mato Grosso do Sul (FUNDECT), por meio da chamada FUNDECT/CAPES n. 02/2017.

\section{REFERÊNCIAS}

ANDERSSON-SKÖLD, Y. et al. A framework for assessing urban greenery's effects and valuing its ecosystem services. Journal of Environmental management, v. 205, p. 274-85, jan. 2018. DOI: https:// doi.org/10.1016/j.jenvman.2017.09.071

ARAGÃO, L. E O. C. The rainforest's water pump. Nature, v. 489, p. 217-8, set. 2012. DOI: 10.1038/ nature11485.

BOLUND, P.; HUNHAMMAR, S. Ecosystem services in urban areas. Ecological Economics, v. 29, n. 2, p. 293301, maio 1999. DOI: https://doi.org/10.1016/S0921-8009(99)00013-0

COSTANZA, R. et al. The value of the world's ecosystem services and natural capital. Nature, v. 387, p. 253-60, maio 1997. DOI: https://doi.org/10.1038/387253a0

COUTTS, C.; HAHN, M. Green infrastructure, ecosystem services, and human health. International Journal of Environmental Research and Public Health , v. 12, n. 8, p. 9768-98, 2015. DOI: 10.3390/ ijerph120809768 
DAVIES, Z. G. et al. Identifying potential sources of variability between vegetation carbon storage estimates for urban areas. Environmental Pollution, v. 183, p. 133-42, dez. 2013. DOI: https://doi.org/10.1016/j. envpol.2013.06.005

DAVIES Z. G. et al. Mapping an urban ecosystem service: quantifying above-ground carbon storage at a city-wide scale. Journal of Applied Ecology, v. 48, n. 5, p. 1125-34, out. 2011. DOI: https://doi.org/10.1111/ j.1365-2664.2011.02021.x

DERKZEN, M. L. et al. Quantifying urban ecosystem services based on high resolution data of urban green space: an assessment for Rotterdam, the Netherland. Journal of Applied Ecology, v. 52, n. 4, p. 1020-32, 2015. DOI: https://doi.org/10.1111/1365-2664.12469

D'ODORICO, P. et al. Ecohydrology of Terrestrial Ecosystems. BioScience, v. 60, n. 11, p. 898-907, dez. 2010. DOI: https://doi.org/10.1525/bio.2010.60.11.6

DORENDORF, J. et al. Both tree and soil carbon need to be quantified for carbon assessments of cities. Urban Forestry \& Urban Greening, v. 14, n. 3, p. 447-55, 2015. DOI: https://doi.org/10.1016/j.ufug.2015.04.005

ELMQVIST, T. et al. Benefits of restoring ecosystem services in urban areas. Current Opinion in Environmental Sustainability, v. 14, p. 101-8, jun. 2015. DOI: https://doi.org/10.1016/j.cosust.2015.05.001

ESCOBEDO, F. J.; KROEGER, T.; WAGNER, J. E. Urban forests and pollution mitigation: Analyzing ecosystem services and disservices. Environmental Pollution, v. 159, n. 8-9, p. 2078-87, ago./set. 2011. DOI: https:// doi.org/10.1016/j.envpol.2011.01.010

GÓMEZ-BAGGETHUN, E.; BARTON, D. Classifying and valuing ecosystem services for urban planning. Ecological Economics, v. 86, p. 235-45, fev. 2013. DOI: https://doi.org/10.1016/j.ecolecon.2012.08.019

HAASE, D. et al. A quantitative review of urban ecosystem service assessments: concepts, models, and implementation. Ambio, a Journal of Environment and Society., v. 43, p. 413-33, maio 2014. DOI: https:// doi.org/10.1007/s13280-014-0504-0

KABISCH, N.; QURESHI, S.; HAASE, D. Human-environment interactions in urban green spaces - a systematic review of contemporary issues and prospects for future research. Environmental Impact Assessment Review, v. 50, p. 25-34, jan. 2015. DOI: https://doi.org/10.1016/j.eiar.2014.08.007

LUEDERITZ, C. et al. A review of urban ecosystem services: six key challenges for future research. Ecosystem Services, v. 14, p. 98-112, ago. 2015. DOI: https://doi.org/10.1016/j.ecoser.2015.05.001

MILLENNIUM ECOSYSTEM ASSESSMENT [MEA]. Ecosystems and human well-being: biodiversity synthesis. Washington, DC: World Resources Institute, Island Press, 2005.

NIEMELÄ, J. et al. Using the ecosystem services approach for better planning and conservation of urban green spaces: a Finland case study. Biodiversity and Conservation, v. 14, v. 19, n. 11, p. 3225-43, out. 2010. DOI: https://doi.org/10.1007/s10531-010-9888-8

NIEMELÄ, J. Ecology of urban green spaces: the way forward in answering major research questions. Landscape and Urban Planning, v. 125, p. 298-303, maio 2014. DOI: https://doi.org/10.1016/j. landurbplan.2013.07.014

NOWAK, D. J. et al. Carbon storage and sequestration by trees in urban and community areas of the United States. Environmental Pollution, v. 178, p. 229-36, jul. 2013. DOI: https://doi.org/10.1016/j. envpol.2013.03.019 
THE ECONOMICS OF ECOSYSTEMS AND BIODIVERSITY [TEEB]. The economics of ecosystems and biodiversity in national and international policy making. Editado por Patrick ten Brink. London; Washington: Earthscan. 2009.

WOLCH, J. R.; BYRNE, J.; NEWELL, J. P. Urban green space, public health, and environmental justice: the challenge of making cities 'just green enough'. Landscape and Urban Planning, v. 125, p. 234-44, maio 2014. DOI: https://doi.org/10.1016/j.landurbplan.2014.01.017

\section{Sobre os autores:}

Fabiany Sampaio Bertucci Tavares: Mestranda em Recursos Naturais com ênfase nos Espaços Verdes Públicos Urbanos pela Universidade Federal de Mato Grosso do Sul (UFMS). Graduada em Arquitetura e Urbanismo pela UFMS. E-mail: fabiany.bertucci@gmail.com, Orcid: http://orcid.org/0000-0002-4890-9155

Ivan Bergier: Doutor em Ciências (Energia Nuclear na Agricultura) pela Universidade de São Paulo (USP). Mestre em Sensoriamento Remoto pelo Instituto Nacional de Pesquisas Espaciais (INPE). Graduado em Ciências Biológicas pela Universidade Federal de São Carlos (UFSCAR). Pesquisador da Embrapa, unidade Pantanal. E-mail: bergiercpap@gmail.com, Orcid: http://orcid.org/0000-0002-1076-8617

Eliane Guaraldo: Pós-doutora pela Pontifícia Universidade Católica de Campinas (PUC Campinas). Doutora, mestre e graduada em Arquitetura e Urbanismo pela Universidade de São Paulo (USP). Professora associada na Universidade Federal de Mato Grosso do Sul (UFMS). Coordenadora do Mestrado em Recursos Naturais da UFMS. E-mail: arq.artes@gmail.com, Orcid: http://orcid.org/0000-0003-2526-1293 
